\title{
La gravidanza in donne con malattia renale policistica autosomica dominante (ADPKD)
}

\author{
Stefania Rastelli, Santina Castellino
}

U.O.C. Nefrologia e Dialisi, P.O. S. Vincenzo, Taormina (ME)

\begin{abstract}
Pregnancy in women with autosomal dominant polycystic Kidney disease (ADPKD)
Abstract. Autosomal dominant polycystic kidney disease (ADPKD) is one of the most common genetic diseases, with an incidence of 1/1,000 live births, and represents the main genetic cause of chronic kidney disease of the adult. A woman with ADPKD that is planning a pregnancy should be aware that both mother and fetus may be susceptible to several risks that must be carefully identified and balanced in time, in order to determine a smooth and conscious pregnancy. The contribution of a multidisciplinary team allows, through recommendations and common lines of thought, to establish an appropriate counseling and improve both maternal and fetal outcomes and nephropathy. This article presents a review of the literature on pregnancy in women with ADPKD, with particular attention to the risks for the mother and the fetus. Finally, an emblematic and successful case of a pregnancy in a woman with ADPKD is reported.
\end{abstract}

Key words: Pregnancy, ADPKD, CKD, Counseling

Conflict of interest: None.

Financial support: None.

Accettato: 25 Febbraio 2014

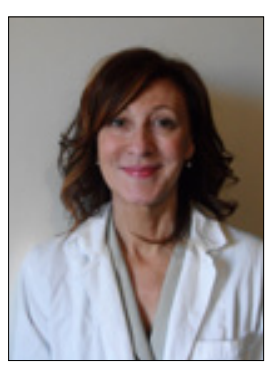

Santina Castellino

\section{Introduzione}

La gravidanza in donne affette da insufficienza renale cronica (IRC) è relativamente poco comune. Per tale motivo, pochi sono gli studi clinici che si sono occupati di questo argomento. Inoltre, i dati della letteratura riguardanti la gravidanza in donne con IRC, data l'impossibilità di condurre studi randomizzati controllati, sono basati soprattutto su studi osservazionali, spesso retrospettivi. Infine, parte di questi studi è datata e non tiene, quindi, conto dei progressi terapeutici intercorsi in questi ultimi decenni. A causa di questo, le raccomandazioni destinate alle donne con IRC sono supportate da deboli evidenze. Tuttavia, grazie alla recente pubblicazione di nuovi studi, tra cui si annoverano anche studi caso-controllo, è stato possibile valutare con maggiore chiarezza i rischi connessi alla gravidanza in presenza di IRC e formulare delle indicazioni basate su evidenze sufficientemente solide (1), grazie alle quali ogni donna affetta da IRC può, oggi, essere informata dei rischi materni e fetali prima di intraprendere una gravidanza e attuare una scelta più consapevole. A oggi, si concorda sul fatto che, nelle donne con IRC, la presenza di un filtrato glomerulare (GFR) $<40 \mathrm{~mL} / \mathrm{min} / 1.73 \mathrm{~m}^{2}$ e di una proteinuria $>1 \mathrm{~g} / 24 \mathrm{~h}$ prima del concepimento o di un'ipertensione non controllata nel corso della gravidanza comporta un aumentato rischio per l'evoluzione della nefropatia materna e per il feto $(2,3)$.

\section{ADPKD e insufficienza renale in gravidanza}

La malattia renale policistica autosomica dominante (ADPKD) rappresenta una delle malattie genetiche più comuni con un'incidenza di 1/1000 nati vivi e costituisce la principale causa genetica di IRC dell'adulto. Il tasso di fertilità negli uomini affetti da rene policistico è comparabile con quello riscontrato in familiari non affetti da tale malattia. L'effetto della gravidanza nelle donne con rene policistico è variabile. La fertilità è nella norma in presenza di una funzione renale normale $(4$, 5). Tuttavia, parallelamente all'aumento di dimensioni delle cisti renali si assiste alla comparsa di ipertensione e al deterioramento della funzione renale, che influenzano negativamente la gravidanza. Oltre a questo, vari sono i fattori che possono incidere sulla funzione renale in questi casi: età della donna, numero di gravidanze, una pregressa ipertensione arteriosa e, soprattutto, una funzione renale già deteriorata (Tab. I). In uno studio longitudinale, Gabow et al. hanno dimostrato che la gravidanza ha effetti negativi sulla funzione renale nelle pluri- 
TABELLA I - FATTORI DI RISCHIO

Fattori di rischio (*pre-gravidanza)

Età donna ( $>30$ anni)

Numero gravidanze $(>3)$

Ipertensione arteriosa"

Creatinina sierica $>1.2 \mathrm{mg} / \mathrm{dL}^{*}$

GFR $<40 \mathrm{~mL} / \mathrm{min} / 1.73 \mathrm{~m}^{2 *}$

Proteinuria $>1 \mathrm{~g} / 24 \mathrm{~h}^{*}$

Infezioni delle vie urinarie

pare con tre o più gravidanze (6). In un altro studio, Chapman et al. hanno dimostrato che, in donne ipertese con rene policistico, il numero di gravidanze ha un lieve ma significativo effetto negativo sulla funzione renale a lungo termine, in particolare nelle donne con quattro o più gravidanze (4). Questi dati sono supportati anche dallo studio di Mohteshamzadeh et al. (7), che mette in relazione il peggioramento della funzione renale con l'età della donna (maggiore di 30 anni) e il numero delle gravidanze (superiore a tre). I dati che riguardano l'outcome fetale nelle donne in gravidanza affette da rene policistico sono, invece, largamente deficitari.

Al fine di valutare le possibili complicanze materne e fetali che si possono presentare in gravidanza, Chapman et al. (4) hanno paragonato donne in gravidanza affette da rene policistico con quelle non affette appartenenti alle stesse famiglie. Le donne affette da rene policistico presentavano una maggiore probabilità di complicanze (in particolare di ipertensione arteriosa, edema e preeclampsia) rispetto a quelle senza rene policistico $(35 \%$ vs $19 \%, \mathrm{P}<0.001)$. La preesistente ipertensione arteriosa era, inoltre, il più importante fattore di rischio per lo sviluppo di complicanze materne e fetali. È stato, infatti, riportato come la preeclampsia si sviluppava nel 54\% delle donne ipertese con rene policistico e solo nell' $8 \%$ delle donne normotese con rene policistico. In accordo con quest'ultimo dato, Milutinovic et al. hanno dimostrato un incremento del rischio di ipertensione indotta dalla gravidanza nelle donne con rene policistico (5). Il tasso di complicanze fetali, invece, era simile tra le donne affette da rene policistico e quelle non affette (32.6\% vs $26.2 \%)$. Tra le donne con rene policistico, la maggior parte delle normotese con creatinina sierica $\leq 1.2 \mathrm{mg} / \mathrm{dL}$ portava a termine la gravidanza senza complicazioni, mentre 1 ' $80 \%$ di quelle con creatinina sierica pre-gravidanza $>1.2 \mathrm{mg} / \mathrm{dL}$ sviluppava insufficienza renale terminale 15 anni prima rispetto alla popolazione generale affetta da rene policistico. Quest'ultimo dato si riferisce, tuttavia, a un campione di solo 5 donne e dovrebbe essere confermato in studi più ampi. Inoltre, è stato riscontrato un aumento, non statisticamente significativo, del rischio di gravidanze ectopiche nelle donne con rene policistico. Tenendo in considerazione che sono state riscontrate alterazioni nell'epitelio degli organi duttali nei pazienti affetti da rene policistico, questo dato potrebbe suggerire che anche l'epitelio dell'ovidotto sia anormale. Infine, a differenza di quanto riportato per lo sviluppo di cisti epatiche (8), non è stato riscontrato un aumento delle cisti renali durante la gravidanza. Questo dato suggerisce che le modifiche ormonali tipiche della gravidanza non dovrebbero promuovere la cistogenesi renale.

\section{ADPKD e ipertensione in gravidanza}

L'ipertensione arteriosa costituisce uno dei sintomi più frequenti nei pazienti affetti da rene policistico, si presenta nel 50-70\% dei pazienti (9), spesso prima della riduzione della funzione renale, e può rappresentare il primo segno della malattia cistica renale. Nella patogenesi dell'ipertensione arteriosa giocano un ruolo fondamentale l'aumento dell'attività del sistema reninaangiotensina (RAS) e l'espansione del volume extracellulare, spesso già presenti agli stadi iniziali della malattia policistica (10). E stato, inoltre, suggerito che l'espansione delle cisti, provocando aree di ischemia renale e incrementando il rilascio di renina, sia largamente responsabile, almeno inizialmente, dell'aumento della pressione arteriosa (11).

L'obiettivo della terapia antipertensiva dovrebbe essere quello di mantenere la pressione arteriosa al di sotto di 130/80 $\mathrm{mmHg}$. Un target terapeutico più rigoroso, al di sotto cioè di $120 / 80 \mathrm{mmHg}$, non è a oggi supportato da sufficienti evidenze, benché sia possibile che il minore livello pressorio possa portare benefici effetti a tutto l'apparato cardiovascolare dei pazienti ipertesi affetti da rene policistico e ipertrofia ventricolare sinistra $(12,13)$. Se i farmaci antipertensivi di prima scelta in pazienti affetti da rene policistico sono classicamente gli ACE-inibitori e gli antagonisti del recettore dell'angiotensina, è noto che, in gravidanza, tali classi sono da evitare, in particolare nelle prime settimane di gestazione, per l'alto rischio fetale (malformazioni, ritardo di crescita, oligo-idramnios, anuria fetale) ad esse associato. Pertanto, le donne in età fertile dovrebbero essere preventivamente informate del rischio per il feto di tali farmaci ed essere trattate con altre classi di farmaci antipertensivi, come i calcioantagonisti, tra cui, in particolare, la nifedipina, che sono considerati la prima scelta nella terapia dell'ipertensione gestazionale.

\section{ADPKD e infezioni in gravidanza}

Circa il 30-50\% dei pazienti con ADPKD durante il corso della vita presenta uno o più episodi infettivi delle vie urinarie. In particolar modo, le donne sono maggiormente soggette rispetto agli uomini allo sviluppo di infezioni sostenute soprattutto da E. Coli ed Enterobacteriaceae. Inoltre, le donne in gravidanza hanno una maggiore predisposizione allo sviluppo di infezioni urinarie (batteriuria asintomatica, cistite acuta e pielonefrite acuta), legata a cambiamenti ormonali, come l'aumentata produzione di progesterone che induce un'ipotonia muscolare e determina una diminuita attività peristaltica dell'uretere, alla compressione uterina che può favorire la comparsa di reflusso vescico-ureterale e, infine, all'aumento di proteine, amminoacidi e glucidi nel filtrato glomerulare con conseguente ridotto potere antibatterico della preurina.

Tra le infezioni urinarie, la più frequente è sicuramente la batteriuria asintomatica, che si presenta nel 2-7\% delle donne gravide (analogamente alle donne non gravide) e nell' $8-14 \%$ delle donne diabetiche (14). La batteriuria asintomatica, a differenza di quanto avviene nelle donne non affette da nefropatie a rischio, nelle quali ha un decorso transitorio e benigno, può, se non trattata adeguatamente, persistere in gravidanza nel $60-85 \%$ dei casi e complicarsi con un quadro di pielone- 
frite acuta nel $15-45 \%$ dei casi. La batteriuria sintomatica è, inoltre, associata a un aumentato rischio di complicanze materne (ipertensione arteriosa gestazionale e preeclampsia) e fetali (aborto, parto pretermine, basso peso alla nascita e mortalità perinatale) (15-17). Fondamentale diventa, quindi, uno screening adeguato per la batteriuria asintomatica, che deve essere eseguito tra la $12^{\mathrm{a}}$ e la $16^{\mathrm{a}}$ settimana di gestazione e, se negativo, deve essere ripetuto all'inizio del $3^{\circ}$ trimestre. Il rischio di complicanza verso la pielonefrite acuta si riduce del $70-80 \%$ quando l'infezione viene eradicata con un trattamento antibiotico standard mirato di 3-7 giorni, con farmaci di prima scelta come i betalattamici (cefalosporine, penicilline), con o senza inibitori delle betalattamasi, e la fosfomicina. Altri antibiotici con un buon profilo di sicurezza in gravidanza sono eritromicina, azitromicina e clindamicina $(1,18)$.

La cistite acuta e la pielonefrite acuta presentano, invece, un'incidenza più bassa, dell' $1-2 \%$ circa ciascuna. La cistite acuta si presenta solitamente con sintomi clinici quali disuria, dolore sovrapubico e, a volte, macroematuria; la pielonefrite ha, invece, un quadro clinico più importante, determinato da sintomi generali, come malessere, brividi, nausea e/o vomito e febbre superiore a $38^{\circ} \mathrm{C}$, e da sintomi locali, come dolore intenso al fianco o pelvico, espressione della flogosi del bacinetto e del parenchima renale, e interessamento dell'angolo costo-vertebrale in assenza o presenza dei sintomi della cistite acuta. Per tale motivo, la gestione di tale evenienza richiede un'ospedalizzazione immediata e il trattamento con antibiotici per via endovenosa.

\section{ADPKD e ingombro addominale in gravidanza}

Un altro problema che riguarda le pazienti con ADPKD è l'effetto massa di reni aumentati di volume a causa della presenza di numerose cisti e/o di un massiccio fegato anch'esso policistico, che potrebbero creare potenzialmente uno spazio inadeguato per la crescita placentare e fetale. L'aumento delle dimensioni renali può, infatti, causare dolore cronico e compressione degli organi adiacenti, con conseguenti inappetenza, reflusso gastroesofageo, malnutrizione e ascite (compressione della vena cava inferiore o della vena porta). Anche se, come già detto, l'ADPKD non pregiudica la fertilità, non è stata a oggi segnalata la gravidanza in pazienti con reni di grandi dimensioni in fase avanzata di insufficienza renale (19).

\section{Caso clinico}

Paziente di anni 31 affetta da rene policistico autosomico dominante (ADPKD) diagnosticato ecograficamente circa 8 anni prima giunge alla nostra osservazione alla nona settimana di gestazione della prima gravidanza. La paziente presenta creatinina sierica di $1.2 \mathrm{mg} / \mathrm{dL}$, GFR pari a $62 \mathrm{~mL} / \mathrm{min}$, ipertensione arteriosa $(130 / 80 \mathrm{mmHg})$ ben controllata con l'uso di ACE-inibitori (enalapril) e storia di infezioni delle vie urinarie, fattori di rischio tali per cui, negli anni precedenti in cui la paziente era stata vista presso altri centri nefrologici, era stata sconsigliata la gravidanza stessa. Al momento della prima visita, per il rischio legato all'uso di ACE-inibitori, viene modificata la terapia antipertensiva e viene introdotta l'alfa metildopa, con un buon controllo della pressione arteriosa nei mesi successivi. Durante le settimane di gestazione, viene, quindi, effettuata un'accurata valutazione clinica che comprende il monitoraggio della pressione arteriosa, del peso corporeo, della diuresi, del bilancio idrico e dell'eventuale presenza di edemi declivi. Viene, inoltre, effettuata una valutazione dei parametri laboratoristici della funzione renale e dei marcatori biologici di preeclampsia (Tab. II). Lo screening per la batteriuria asintomatica viene eseguito tra la $12^{\text {a }}$ e la $16^{\text {a }}$ settimana. Per il riscontro di un'infezione da $E$. Coli, si intraprende un ciclo di antibiotici per via orale, ripetuto successivamente per un secondo episodio infettivo. Nei mesi successivi la crescita del feto procede normalmente; entrambi i reni non presentano dimensioni tali da mettere a rischio lo sviluppo fetale e placentare, anche per la concomitante assenza di lesioni cistiche epatiche. La gravidanza procede senza alcuna complicazione fino alla $34^{\mathrm{a}}$ settimana, epoca in cui la paziente presenta un quadro di preeclampsia con rialzo dei valori pressori fino a 160/90 $\mathrm{mmHg}$, comparsa di proteinuria al di sopra di $2 \mathrm{~g} / 24 \mathrm{~h}$, peggioramento della funzione renale con un aumento della creatinina sierica fino a $1.4 \mathrm{mg} / \mathrm{dL}$ e modesta riduzione delle piastrine. Viene, pertanto, indotto il parto con nascita di un neonato di $2 \mathrm{Kg}$, seguito, nelle settimane successive, per la prematurità, presso l'unità di terapia intensiva neonatale, da cui è stato poi dimesso senza alcuna sequela. Anche la madre viene dimessa in seguito alla stabilizzazione clinica, con valori di creatinina di $1.4 \mathrm{mg} / \mathrm{dL}$ e valori pressori pre-gravidanza, con assunzione di ACE-inibitori, e, successivamente, viene affidata al nefrologo di riferimento.

TABELLA II - VALUTAZIONE CLINICA IN PRE-GRAVIDANZA E IN GRAVIDANZA

Valutazione in pre-gravidanza

Infezioni urinarie ricorrenti

Ipertensione arteriosa

Presenza di ematuria e cilindruria al sedimento

Presenza di microalbuminuria o proteinuria

Insufficienza renale

Anomalie ecografiche

Familiarità per malattie renali
Valutazione in gravidanza

Monitoraggio pressione arteriosa

Monitoraggio peso corporeo, diuresi, bilancio idrico

Presenza di edemi declivi

Esame di laboratorio (esame urine, proteinuria, albuminuria, uricemia)

Funzione renale

Marcatori biologici preeclampsia

Ecografia apparato urinario 


\section{Conclusioni}

La valutazione di donne affette da ADPKD in gravidanza è un ambito che richiede un confronto, non solo tra nefrologi che si trovano ad affrontare tali problematiche, ma anche con altri specialisti come l'ostetrico, il neonatologo e il genetista. Infatti, nel caso di malattie ereditarie come l'ADPKD il ruolo del genetista è di fondamentale importanza, in quanto una diagnosi genetica prenatale è determinante per una scelta più serena e consapevole della gravidanza. L'apporto del team multidisciplinare, utilizzando raccomandazioni e percorsi condivisi, consente di instaurare un opportuno counseling e di migliorare gli outcome sia materni, che fetali che della nefropatia.

\section{Riassunto}

La malattia renale policistica autosomica dominante (ADPKD) rappresenta una delle malattie genetiche più comuni con un'incidenza di 1/1000 nati vivi e, costituisce la principale causa genetica di IRC dell'adulto. Quando una donna affetta da ADPKD pianifica una gravidanza, sia la madre che il feto possono andare in contro a rischi che devono essere attentamente identificati e soppesati per tempo al fine di determinare una scelta più serena e consapevole della gravidanza stessa. L'apporto del team multidisciplina- re, utilizzando raccomandazioni e percorsi condivisi, consente di instaurare un opportuno counseling e di migliorare gli outcomes sia materni che fetali e della nefropatia. In questo articolo viene presentata una rassegna della letteratura sulla gravidanza in donne con ADPKD, con particolare attenzione ai rischi per la madre e per il nascituro. Infine, viene riportato un caso clinico emblematico di una gravidanza in una donna con ADPKD risoltosi positivamente.

Parole chiave: Gravidanza, ADPKD, IRC, Counseling

Dichiarazione di conflitto di interessi: Gli Autori dichiarano di non avere conflitto di interesse.

Contributi economici agli Autori: Gli Autori dichiarano di non aver ricevuto sponsorizzazioni economiche per la preparazione dell'articolo.

Indirizzo degli Autori:

Dr.ssa Santina Castellino

U.O.C. Nefrologia e Dialisi

P.O. "S. Vincenzo"

Contrada Sirina, Taormina (ME)

sancas@tin.it

\section{Bibliografia}

1. Rene e Gravidanza: Suggerimenti di pratica clinica e di applicazione delle Linee Guida. Nephromeet (accesso il 23 Febbraio 2014).

2. Davison JM, Lindheimer MD. Pregnancy and chronic kidney disease. Semin Nephrol 2011; 31 (1): 86-99.

3. Imbasciati E, Gregorini G, Cabiddu G, et al. Pregnancy in CKD stages 3 to 5: fetal and maternal outcomes. Am J Kidney Dis 2007; 49 (6): 753-62.

4. Chapman AB, Johnson AM, Gabow PA. Pregnancy outcome and its relationship to progression of renal failure in autosomal dominant polycystic kidney disease. J Am Soc Nephrol 1994; 5 (5): 1178-85.

5. Milutinovic J, Fialkow P, Agodoa L, Phillips LA, Bryant JI. Fertility and pregnancy complications in women with autosomal dominant polycystic kidney disease. Obstet Gynecol 1985; 61: 566-70.

6. Gabow PA, Johnson AM, Kaehny WD, et al. Factors affecting the progression of renal disease in autosomal dominant polycystic kidney disease. Kidney Int 1992; 41: 1311-9.

7. Mohteshamzadeh M, Coutinho A, Erekosima I, Rustom R, Wong CF. Successful pregnancy in a patient with Landesman's Group $\mathrm{C}$ autosomal dominant polycystic kidney disease. Nat Clin Pract Nephrol 2008; 4 (4): 227-31.

8. Gabow PA, Johnson AM, Kaehny WD, Manco-Johnson ML, Duley IT, Everson GT. Risk factors for the development of hepatic cysts in autosomal dominant polycystic kidney disease. Hepatology 1990; 11 (6): 1033-7.

9. Ecder T, Schrier RW. Hypertension in autosomal-dominant polycystic kidney disease: early occurrence and unique aspects. J Am Soc Nephrol 2001; 12 (1): 194-200.

10. Barrett BJ, Foley R, Morgan J, Hefferton D, Parfrey P. Differences in hormonal and renal vascular responses between normotensive patients with autosomal dominant polycystic kidney disease and unaffected family members. Kidney Int 1994; 46 (4): 1118-23.

11. Chapman AB, Johnson A, Gabow PA, Schrier RW. The renin-angiotensin-aldosterone system and autosomal dominant polycystic kidney disease. N Engl J Med 1990; 323: 1091-6.

12. Chapman AB, Torres VE, Perrone RD, et al. The HALT polycystic kidney disease trials: design and implementation. Clin J Am Soc Nephrol 2010; 5: 102-9.

13. Kidney Disease Outcomes Quality Initiative (K/DOQI). K/DOQI clinical practice guidelines on hypertension and antihypertensive agents in chronic kidney disease. Am J Kidney Dis 2004; 43: S1. [

14. Nicolle LE, Bradley S, Colgan R, Rice JC, Schaeffer A, Hooton TH. Infectious Diseases Society of America Guidelines for the Diagnosis and Treatment of Asymptomatic Bacteriuria in Adults. Clin Infect Dis 2005; 40 (5): 643-54.

15. Mittendorf R, Williams MA, Kass EH. Prevention of preterm delivery and low birth weight associated with asymptomatic bacteriuria. Clin Infect Dis 1992; 14 (4): 927-32.

16. Millar LK, Cox SM. Urinary tract infections complicating pregnancy. Infect Dis Clin North Am 1997; 11 (1): 13-26.

17. Sheiner E, Mazor-Drey E, Levy A. Asymptomatic bacteriuria during pregnancy. J Matern Fetal Neonatal Med 2009; 22 (5): 423-7.

18. Guinto VT, De Guia B, Festin MR, Dowswell T. Different antibiotic regimens for treating asymptomatic bacteriuria in pregnancy. Cochrane Database Syst Rev 2010; (9): CD007855.

19. Jung JH, Kim MJ, Lim HJ, et al. Successful Pregnancy in a Patient with Autosomal Dominant Polycystic Kidney Disease on LongTerm Hemodialysis. J Korean Med Sci 2014; 29: 301-4. 